Original Research

\title{
Smartphone Usage at Bedtime: The Effect of Sleep-Smartphone Hygiene, Trait Anxiety, and FOMO on Sleep Quality
}

\author{
Dorit Shoval, Nama Tal, Orna Tzischinsky *
}

Educational Counselling Department, The Max Stern Yezreel Valley College, Emek Yezreel, Israel; EMails: dorith@yvc.ac.il; nammatal@hotmail.com; orna3007@gmail.com

* Correspondence: Orna Tzischinsky; E-Mail: Orna3007@gmail.com

Academic Editor: Luigi De Gennaro

OBM Neurobiology

2021, volume 5, issue 1

doi:10.21926/obm.neurobiol.2101088
Received: July 21, 2020

Accepted: February 19, 2021

Published: March 08, 2021

\begin{abstract}
The present study was aimed to examine the relationship of sleep-smartphone hygiene, i.e., the habits of smartphone usage in the sleep environment, with the sleep quality among students and whether this relationship could be explained through psychological factors. A total of 467 Israeli college students were investigated using five questionnaires, namely, Sleep-Smartphone Hygiene Questionnaire (SSHQ), Fear of Missing Out Scale (FoMOs), Trait Anxiety Inventory (STAI-T), Pittsburgh Sleep Quality Index (PSQI), and a demographic questionnaire. The analysis of the collected data revealed positive Pearson's correlations among sleep-smartphone hygiene, trait anxiety, FOMO, and sleep quality. The moderation model was observed to be significant, indicating that sleep quality could be predicted on the basis of sleep-smartphone hygiene, trait anxiety, and FOMO, which together explained $20 \%$ of the variance in the sleep quality. Trait anxiety was determined to be a moderating variable for the relationship between sleep-smartphone hygiene and sleep quality. These findings have important implications in understanding the effect of smartphone usage on sleep quality and developing an evidence-based intervention program for the students to cope with the impairment of sleep quality. It is recommended to design interventions that would address both behavioral change and the intrapersonal aspects such as trait anxiety.
\end{abstract}

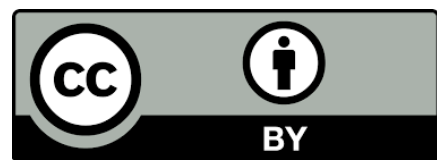

(C) 2021 by the author. This is an open access article distributed under the conditions of the Creative Commons by Attribution License, which permits unrestricted use, distribution, and reproduction in any medium or format, provided the original work is correctly cited. 


\section{Keywords}

Smartphone; sleep hygiene; fear of missing out (FOMO); trait anxiety; sleep quality

\section{Introduction}

The usage of mobile information and communication technologies such as smartphones has accelerated in recent years [1]. According to a previous study, the phone companies shipped a total of 347.4 million smartphones across the world in the first quarter of 2017 alone [2]. Smartphones are popular devices that are capable of processing more information compared to all the other technologies. The various features offered by smartphones include games, access to the internet and social networks, messaging, videos, multimedia, and navigation, besides the most basic feature of communication [3]. The accelerated usage of smartphones in recent years and the stupendous number of features offered by these phones have raised concerns regarding the effect of smartphones on the health of their users [4]. Numerous adverse health outcomes associated with high smartphone usage have been identified, including obesity, decreased physical activity [5], and reduced well-being, which are reflected in the increased levels of anxiety and depression $[6,7]$ and poor sleep quality $[1,5]$.

The present study was aimed to explore the relationship between general smartphone usage, particularly at bedtime, and sleep quality among college students. Previous studies have reported distinctly different impacts of high and low smartphone usage on sleep quality in terms of sleep disturbance and daytime dysfunction [3] or in terms of sleep onset latency and bedtime [8]. All such studies reported poor sleep quality among the students (adolescents as well as young adults) who are high-intensity smartphone users.

It is common for college students to have a poor sleep and inconsistent sleep schedules, with nearly $60 \%$ of them complaining of poor sleep quality and close to $70 \%$ reporting sleep disordersproblems [9]. Insufficient sleep duration is reported to occur in epidemic proportions among college students [10], resulting in a substantial impact on their physical as well as emotional well-being [11]. The various factors leading to insufficient sleep and irregular sleep schedules are, in part, social and academic stress, long and hectic work hours, freedom from parental supervision, and extensive consumption of tobacco, alcohol, and drugs, among others [11, 12]. All these factors cause an increase in the behaviors that impair sleep quality and are collectively designated as poor sleep hygiene. Sleep hygiene is defined as a set of behaviors and environmental variables associated with the promotion of proper sleep [13], including modifiable environmental factors (such as a conducive sleep environment), scheduling (for example, a consistent sleep-wake schedule), sleep practices (including a predictable bedtime routine), and physiological factors (such as reduced caffeine consumption) [14, 15]. Poor sleep hygiene practices, including the increased usage of technology (such as a computer, smartphone, tablet, etc.) are reported to increase the risk of sleep problems among college students $[14,16]$.

In the present study, the usage of technology in the sleep environment [and during sleep time] is referred to as sleep-smartphone hygiene (SSH). SSH, in definition, refers to the behavioral traits of smartphone usage at bedtime in the sleep environment (such as sleeping with the smartphone next to the bed, using a smartphone while in bed prior to sleeping), in the middle of the night (such 
as using the smartphone during the night and responding to the stimuli from various applications, such as email, Facebook, or Instagram), or in the early morning (such as using the smartphone as an alarm clock, checking the smartphone just after waking). The literature reports that students, in general, have poor sleep hygiene, based on which the first hypothesis of the present study was formulated, which was that the student participants would report a high prevalence and high intensity of the behaviors characterized by increased smartphone usage in the sleep environment (i.e., they would report a poor SSH). The second hypothesis was based on the earlier findings of the presence of smartphones in the bedroom (specifically at bedtime) affecting sleep [1, 8, 17], and accordingly, an association between poor SSH and reduced sleep quality was hypothesized.

In a recent study, Rogers and Barber [16] examined the effect of an educational intervention on the usage of sleep-disruptive technology and sleep hygiene, and no direct effects were reported, which raised questions regarding the mechanisms operating behind the association between SSH and sleep quality. The present study was aimed to address this concern by exploring the moderate effect of psychological factors on this association. The literature highlights two possible psychological factors in this regard: trait anxiety and FOMO (fear of missing out). Trait anxiety refers to a tendency to experience negative emotions, such as fears, worries, and anxieties, in numerous situations. It manifests as repeated fears and even physiological symptoms. Two levels of trait anxiety could be considered: the perceptual level, at which there is a tendency to attend closely to threatening stimuli, and the cognitive level, at which the tendency is to interpret stimuli in a threatening manner [18]. Studies have reported an association between trait anxiety and poor sleep [19]. On the other hand, FOMO is a general state of anxiety associated with missing out on rewarding experiences - a fear that is often the driving force behind social media engagement [20]. Individuals with FOMO report feeling disconnected and missing out on "something" in the absence of access to online communication and, therefore, prefer having their smartphones within reach even at night [21]. Such feelings render it quite difficult for the highly-invested users to disengage from social media even at bedtime [18].

In this context, the present study proposes a theoretical moderation model, which asserts that psychological factors (such as trait anxiety and FOMO) serve as moderators between the behavioral habits of smartphone usage in the sleep environment and the individual's sleep quality (refer to Figure 1). 


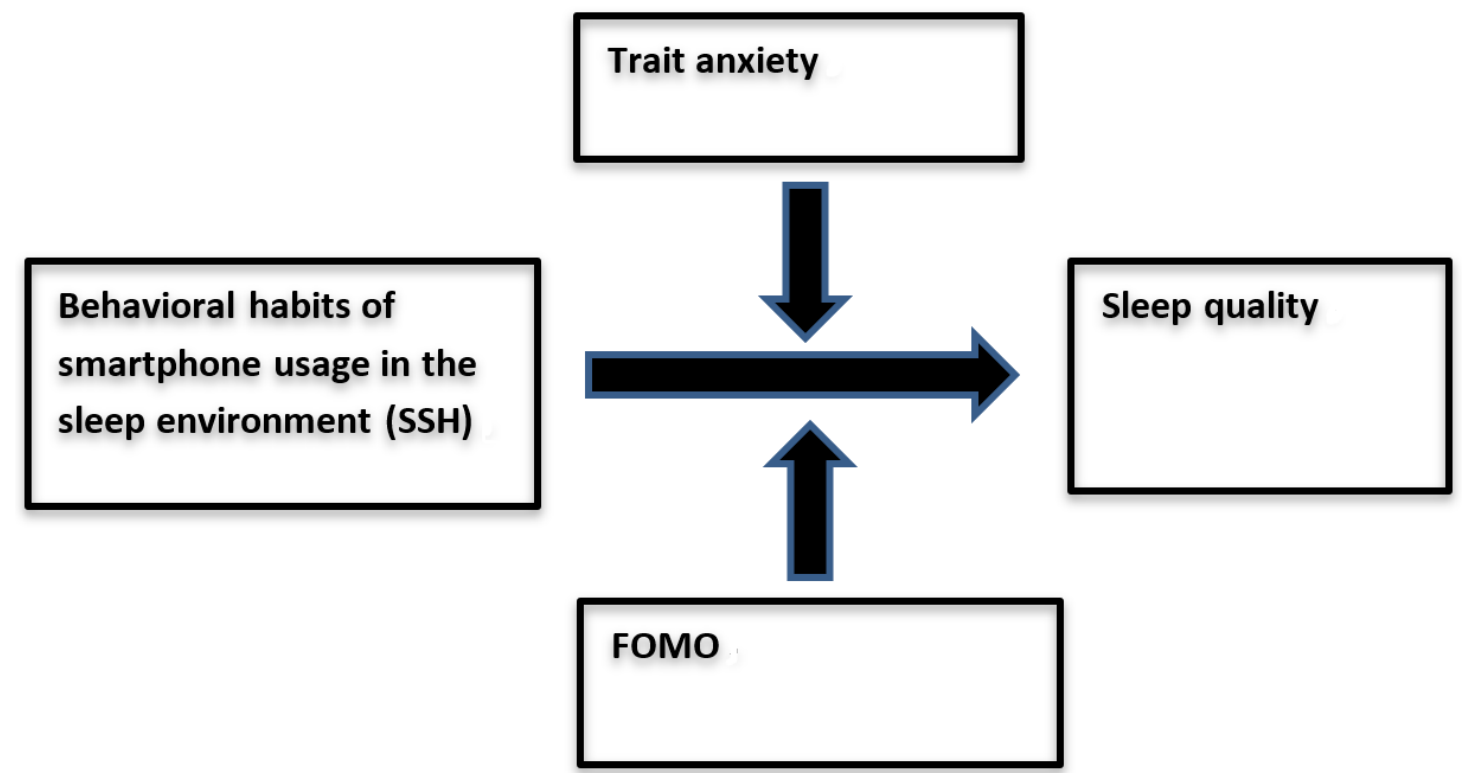

Figure 1 The proposed moderation model for the association between $\mathrm{SSH}$ and sleep quality.

\section{Ethics Statement}

The complete protocol of the present study was approved by the College Institutional Review Board (EMEK-YVC 2019-15). The completion of the questionnaires was voluntary, and the respondents were informed that they could withdraw their participation from the study at any point if they wished to.

\section{Materials and Methods}

\subsection{Participants}

The study participants were 467 Israeli college students (316 women and 141 men; age 19-30 years; $M_{\text {age }}=25$ years, $S D_{\text {age }}=2.69$ years) . The inclusion criterion for participation in the present study was to be a student without any health problems, while the exclusion criterion was to be a student who was a parent or pregnant. The questionnaires completed by three participants were excluded from the final analysis.

\subsection{Tools}

\subsubsection{Demographic Questionnaire}

The participants were requested to provide information regarding their age and gender by means of this questionnaire.

\subsubsection{Sleep-Smartphone Hygiene Questionnaire (SSHQ)}

The SSHQ is a Likert-type scale containing ten items, which are an elaboration of the two items in the Sleep Hygiene Index, with scores for each item ranging from 1 (never) to 5 (always) [22]. The ten items explore the habits associated with smartphone usage in the sleep environment prior to 
bedtime, in the middle of the night, or in the early morning (such as "I sleep with my smartphone in the bedroom", "I scroll through my smartphone while in bed before I fall asleep", and "I check my smartphone during the night"). The fourth item in the SSHQ involved reverse coding, and its score was, therefore, reversed prior to calculating the total score. The scores obtained for all ten items in the scale were averaged to obtain the final SSH score for each participant, with a high score indicating high prevalence and high intensity of the behaviors characterized by increased smartphone usage in the bedroom environment (i.e., indicating a poor SSH). Content validity for the SSHQ was established through a process of expert feedback and revision which included: (1) development of an initial item set; (2) evaluation of the initial item set by an expert panel (students and sleep experts); (3) revision of the initial item set based on evaluation and feedback; and (4) a final expert evaluation of the revised items. Cronbach's alpha reliability coefficient for the current sample was determined to be 0.71 .

\subsubsection{The Fear of Missing Out Scale (FoMOs) [20]}

The FoMOs contains ten items (such as "I get anxious when I do not know what my friends are up to") on a 5-point Likert-type scale, with scores for each item ranging from 1 (not at all true for me) to 5 (extremely true for me). The scores of all ten items were averaged to generate the overall score ranging from 1 to 5 . Cronbach's alpha reliability coefficient for the current sample was 0.85 .

\subsubsection{The State-Trait Anxiety Inventory (STAI-T) [23]}

STAI-T is a 20-item inventory that measures trait anxiety as a psychological construct involving self-perception, the higher-order factors of negative emotional experience, and the sub-factors of depression and anxiety. Each item is weighted equally on a 4-point scale, with scores for each item ranging from 1 (not at all true for me) to 3 (extremely true for me). The potential scores for the STAIT range from 20 to 80 . Cronbach's alpha reliability coefficient for the present sample was 0.93 .

\subsubsection{Pittsburgh Sleep Quality Index (PSQI) [24]}

The PSQI is a self-reporting questionnaire that assesses sleep quality and sleep disturbance over a one-month period. It contains 19 items categorized into seven equally-weighted subscales, namely, subjective sleep quality, sleep latency, sleep duration, habitual sleep efficiency, sleep disturbances, use of sleep medication, and daytime dysfunction, on a 4-point scale of 0 to 3 ( $0=$ no difficulty; $3=$ severe difficulty). The scores of all subscales are summed up to generate a global PSQI score ranging from 0 to 21, with the higher scores indicating worse sleep quality. In the present study, only the total PSQI score was used, which provides an overall summary of the participants' sleep experience and sleep quality during the previous two weeks, with the higher scores indicating poorer sleep quality. A total score of over 5 implies the presence of sleep disturbances. Cronbach's alpha reliability coefficient for the seven subscale scores was 0.73 .

\subsection{Procedure}

After obtaining the approval for the present research from the college's ethics committee, the participants for the study were recruited on social networks and the college campus. The participants completed the online questionnaires anonymously using Google Forms. All the 
questionnaires were translated from English to Hebrew by a person, and the completed questionnaires were translated back to English by a different person.

\subsection{Data Analysis}

The assumptions regarding normal distribution for all the study variables were verified via skewness and kurtosis with values ranging between -1 and +1 considered to fulfill the requirements for using the parametric testing methods [25]. The means, standard deviations, and Pearson's correlations among the study variables were analyzed. The differences between the male and female students were analyzed using $t$-tests. These statistical analyses were performed to ensure, prior to running the statistical model, that the variance in these variables did not exert any effect on the dependent variable. The proposed model was evaluated by conducting a moderation analysis using SPSS 24 (IBM, Chicago, IL, USA) with the PROCESS macro Model 2 [26]. SSHQ was used as the independent variable, and sleep quality (PSQI) was used as the dependent variable. Trait anxiety and FOMO were the potential moderators, and age was used as the covariate. Moderation is considered to have occurred when the interaction between the independent variable and the potential moderator is statistically significant.

\subsubsection{Sample Size Considerations}

A sample size of 425 students was required to allow the evaluation of the proposed ANCOVA model (three independent variables plus two interaction effects) with the inclusion of a maximum of two covariates at $95 \%$ confidence and $80 \%$ power, assuming a small-to-medium effect size $(f=$ 0.175 ). With the assumption that $10 \%$ of the questionnaires would be unusable, a total of 468 students were recruited. GPower program was used for computing the sample size [27].

\section{Results}

Table 1 The means, standard deviations, and ranges for each variable.

\begin{tabular}{lccccc}
\hline Variable & Mean (SD) & Min & Max & Skewness & Kurtosis \\
\hline Smartphone-sleep hygiene (SSHQ) & $3.82(0.60)$ & 1.8 & 5.0 & -0.52 & 0.06 \\
Fear of missing out (FoMOs) & $2.59(0.70)$ & 1.0 & 4.9 & 0.29 & -0.13 \\
Trait anxiety (STAI-T) & $39.75(11.80)$ & 20 & 77 & 0.63 & -0.8 \\
Sleep quality (PSQI) & $5.21(2.80)$ & 1 & 17 & 0.84 & 0.91 \\
\hline
\end{tabular}

\subsection{SSHQ}

According to the first hypothesis, the students were expected to report poor SSH habits of smartphone usage in the sleep environment. Table 2 presents the frequency (\%) of each response to every question. 
Table 2 Frequency (\%) of each response to every question in the SSHQ*.

\begin{tabular}{|c|c|c|c|c|c|c|}
\hline & & Never & Rarely & Sometimes & Often & Always \\
\hline 1 & Sleeps with smartphone in the bedroom & 1.7 & 1.5 & 1.3 & 4.8 & 90.7 \\
\hline 2 & Sleeps with smartphone next to the bed & 7.6 & 6.1 & 8.1 & 14.1 & 63.6 \\
\hline 3 & Leaves smartphone on overnight & 1.3 & 1.7 & 2.6 & 5.4 & 88.9 \\
\hline $4^{\mathrm{R}}$ & Switches smartphone to silent mode at night & 23.6 & 11.3 & 15 & 9.5 & 40.6 \\
\hline 5 & Scrolls through smartphone in bed before going to sleep & 3.5 & 8.5 & 14.8 & 23 & 50.0 \\
\hline 6 & $\begin{array}{l}\text { Scrolls through smartphone in bed after turning off the } \\
\text { lights (having already planned to go to sleep) }\end{array}$ & 10.2 & 14.5 & 18 & 24.5 & 32.8 \\
\hline 7 & Checks smartphone during the night & 20.8 & 23.0 & 21.5 & 18.7 & 16.1 \\
\hline 8 & $\begin{array}{l}\text { Responds to one of the following applications: } \\
\text { email/Facebook/Instagram during the night }\end{array}$ & 34.5 & 26.9 & 21.5 & 8.2 & 8.9 \\
\hline 9 & Uses smartphone as an alarm clock & 9.0 & 1.7 & 1.7 & 4.3 & 91.3 \\
\hline 10 & Checks smartphone first thing on waking up & 2.2 & 4.8 & 16.7 & 29.5 & 46.9 \\
\hline
\end{tabular}

Note. ${ }^{*}$ The mode response to each of the items is presented in bold font.

As indicated in Table 2, the students reported poor SSH. Most of the students (90.7\%) reported sleeping along with their smartphones inside their bedrooms, with $88.9 \%$ of the students having the smartphones switched on throughout the night, $50 \%$ of the students scrolling through the smartphones while in bed prior to falling asleep, and $32.8 \%$ of the students scrolling through the smartphones after turning off the lights. While $43.8 \%$ of the students reported having "never used" or "rarely used" their smartphones during the night, $34.8 \%$ reported that they "often" or "always" check their smartphones during the night. In addition, $76.4 \%$ of the students reported that checking their smartphone was the first thing they do upon waking up.

\subsection{The Moderation Model}

No statistically significant difference was observed between the genders in terms of sleep quality, as reflected in the total score of the PSQI $\left(t_{(459)}=0.6\right.$, ns.). Therefore, all participants were subsequently treated as a single group. Pearson's correlations among the study variables were consistent with the expected associations based on the proposed model; SSH, FOMO, and trait anxiety correlated positively with sleep quality (Table 3 ). 
Table 3 Pearson's correlations among the study variables.

\begin{tabular}{llll}
\hline & $\begin{array}{l}\text { Sleep-smartphone hygiene } \\
\text { (SSHQ) }\end{array}$ & $\begin{array}{l}\text { Fear of missing out } \\
\text { (FoMOs) }\end{array}$ & $\begin{array}{l}\text { Trait anxiety } \\
\text { (STAI-T) }\end{array}$ \\
\hline $\begin{array}{l}\text { Fear of missing out } \\
\text { (FoMOs) }\end{array}$ & $0.15^{* *}$ & & \\
$\begin{array}{l}\text { Trait anxiety } \\
\text { (STAI-T) }\end{array}$ & $0.14^{* *}$ & $0.51^{* *}$ & \\
Sleep quality (PSQI) & $0.17^{* *}$ & & $0.43^{* *}$ \\
\hline
\end{tabular}

Note. ${ }^{* *} \mathrm{p}<.01$ after Bonferroni adjustment.

A multiple regression test was performed to predict the sleep quality (PSQI) based on SSHQ, STAI$\mathrm{T}$, and FoMOs, and the main findings are presented in Table 4.

Table 4 Multiple regression for sleep quality as a function of SSH, trait anxiety, and FOMO.

\begin{tabular}{lllll}
\hline Predictive variable & $\mathrm{B}$ & $t$ & $\mathrm{R}^{2}$ & $F_{(3,457)}$ \\
\hline Trait anxiety & 0.408 & $8.53 * * *$ & 0.20 & $29.59 * * *$ \\
FOMO & 0.024 & 0.50 & & \\
Sleep-smartphone hygiene (SSH) & 0.108 & $2.52 * *$ & & \\
\hline
\end{tabular}

Note. ${ }^{* *} p<.005,{ }^{* * *} p<.001$.

As indicated in Table 4, the regression model was significant $\left(F_{[4,456]}=29.59, p<0.001\right)$, i.e., the sleep quality (measured in terms of the PSQI) could be predicted on the basis of trait anxiety (measured using STAI-T), FOMO (measured using the FoMOs), and SSH (measured using the SSHQ), and these three variables explained $20 \%$ of the total variance in the sleep quality. Table 4 also reveals that the trait anxiety variable offered the highest unique contribution $(\beta=0.45, p<0.001)$, followed by SSH (SSHQ; $\beta=0.12, p<0.005$ ). On the contrary, the contribution of FOMO to the prediction of sleep quality was not significant $(\beta=-0.01, \beta=0.06, \mathrm{~ns})$.

On the basis of these findings, a model that included SSH as the independent variable and sleep quality as the dependent variable was evaluated, considering psychological factors (trait anxiety and FOMO) as the moderators and age as the covariate. It was revealed that the model was significant $\left(F_{(5,455)}=24.92, p<0.001\right)$ and explained $21.5 \%$ of the variance. The correlation between SSH and sleep quality was observed to be positive and significant $(b=0.6587, S E=0.2070, p<0.002)$ and conditional on the mean anxiety (mean-centered anxiety $=0$ ). The correlation between trait anxiety and sleep quality was also positive and significant and conditional on mean SSH $(b=0.1076, \mathrm{SE}=$ $0.0120, p<0.001)$. Trait anxiety was observed to be a statistically significant moderator of the relationship between SSH and sleep quality $(F(1,455)=5.24, p<0.02)$. The interaction between SSH and trait anxiety explained $0.9 \%$ of the variance in sleep quality. At low levels of trait anxiety $(-1$ $\mathrm{SD})$, the relationship between the sleep quality and the SSH was positive although insignificant. At the median and high levels of trait anxiety, the relationship between the sleep quality and the SSH was positive and also significant. FOMO did not exert a statistically significant effect $(t(455)=-1.21$, 
$p>0.23$ ) on sleep quality (conditional on the mean anxiety) and was, therefore, not a moderator of the relationship between SSH and sleep quality $(F(1,455)=1.72, p>0.19)$.

\section{Discussion}

The present study was aimed to examine the association of the behavioral habits of smartphone usage in the sleep environment (SSH) with the sleep quality among college students and explore whether the psychological factors moderate this association. The results partially confirmed the research hypotheses formulated in the present study. It was revealed that these habits were indeed associated with sleep quality and that this association was moderated by the psychological factor trait anxiety, although not by the other one, i.e., FOMO.

\subsection{SSH Behavioral Habits in the Sleep Environment}

SSH refers to the behaviors such as using a smartphone prior to bedtime, during the night, and in the early morning after waking up $[13,28]$. The first hypothesis of the present study was that the students would report a poor SSH, i.e., a high prevalence and high intensity of the behaviors characterized by increased smartphone usage in the bedroom. This hypothesis was confirmed by the overall mean of the SSHQ index, which revealed that most of the participants reported this behavior. In addition, an individual examination of each of the items in the combined SSHQ index revealed that in all items, the students had reported behavioral habits that reflected a poor SSH. Most of the students reported sleeping along with their smartphone inside the bedroom, close to their bed, and using the smartphone while in bed prior to falling asleep. Moreover, most students reported that checking their smartphones was the first thing they did after they woke up. The percentage of the students reporting smartphone usage during the night was low.

The findings of the present study were consistent with those of the other previously-reported studies that examined the SSH behavioral habits among the students [16, 29, 30]. For instance, in a study examining student athletes [31], 70.2\% of the participants reported scrolling through their smartphones after switching off the lights. The findings of this, as well as other published studies, reveal that students demonstrate a poor $\mathrm{SSH}$.

\subsection{The Moderation Model}

The moderation model proposed in the present study, as stated earlier, utilized psychological factors (trait anxiety and FOMO) to moderate the relationship between the SSH behavioral habits and sleep quality.

As expected, the correlation among the study variables was significant although weak. This weak correlation could be explained by the difference between the measurements of sleep-related behaviors and those of sleep-related attitudes and feelings [32]. However, this finding further reinforces the important role of the moderation model in explaining the dynamics of the association among the variables; and indeed, the model explained $21.5 \%$ of the total variance in the sleep quality. A positive relationship was observed between the SSH and the sleep quality, which was significantly conditioned by trait anxiety and not by FOMO. The interaction between the SSH and the trait anxiety explained $0.9 \%$ of the variance in the sleep quality. At the median and high levels of trait anxiety, the association between the sleep quality and the SSH was positive and significant. 
In contrast to the second hypothesis of the present study, FOMO was not a significant moderator. It could be that FOMO characterizes the adolescents more than the young adult student population that was examined in the present study. Owing to the characteristics of life in Israel, most of the undergraduate students in Israel are older compared to their counterparts in the United States or Europe, and are, to a large extent, far from adolescence. Adolescences are known to use social networks much more than young adults for communicating with their peers, expressing their belonging, self-assertion, popularity, and seeking social support. The social media applications target these adolescents and encourage them to remain online constantly, and this intensive usage of social networks becomes a source of stress accompanying by FOMO [33]. Therefore, it is recommended to extend the present study with the inclusion of the adolescent population in future research.

Since all the SSH measures demonstrated an intense usage of smartphones in the sleep environment, it is reasonable to assume that the impairment of sleep quality cannot be explained by these behavioral habits alone. The fact that a psychological factor, named trait anxiety, was observed to moderate the relationship between behavioral habits and sleep quality is important. The presence of a smartphone inside the bedroom does not necessarily affect the sleep quality, although sleep quality is impaired in the case where the individuals are afflicted with trait anxiety and also keep their smartphones close to their beds. This finding is consistent with those of the other studies that have previously reported trait anxiety $[3,18]$ to be associated with intense smartphone usage in the sleep environment and with various other measures of sleep [8].

\subsection{Limitations}

Although the present study revealed certain important findings, it also has certain limitations. First, the results were based on self-reporting questionnaires translated to Hebrew for comprehension by the students. Previous studies have already discussed the gap between the subjective reporting of sleep quality and its objective measurement [12]. Moreover, the study was conducted by means of a web-based survey, which is subject to self-selection. Therefore, it is recommended that future studies employ objective measurements (actigraphy) to assess sleep quality. Second, the study employed a new questionnaire for measuring the $\mathrm{SSH}$, and even though the content validation process for the questionnaire was adequate, further research is required to establish its validity. Third, the present study did not address the diversity of the participants. The students from different locations and social structures, such as different race/ethnicity, nativity status, and social class, might exhibit different SSH habits, and their sleep quality may vary accordingly. Future studies should explore whether individual circumstances and social locations of the participants serve as risk factors or protective and resilience factors for sleep quality.

\section{Conclusions}

The findings of the present study have important implications for understanding the effect of smartphone usage on sleep quality and, accordingly, develop an evidence-based intervention program for students. An understanding of the association between the SSH and the sleep quality among students would be beneficial to those seeking effective practices to cope with the impairment of sleep quality. The recommendations for behavioral change suggested in certain educational programs encourage sleep hygiene in regard to smartphones [34], although this alone 
would not be sufficient as simply removing the smartphones out of the bedroom would not necessarily improve sleep quality. Therefore, it is emphasized that the recommendations should also address intrapersonal aspects, such as trait anxiety, and should, accordingly, incorporate behavioral changes that encourage a hygienic sleep environment (which, of course, also includes removing the smartphones from the bedroom) and the techniques for reducing anxiety such as CBT [35] or mindfulness [36].

It is noteworthy that the concept of sleep-smartphone hygiene (SSH) is fairly recent. In the present study, SSH was used as a continuous variable, considering that a high score would reflect a high prevalence and a high intensity of smartphone usage in the sleep environment. However, it is important to continue investigating whether it is possible to identify the subgroup that has a poor $\mathrm{SSH}$ and whether the characteristics of this subgroup are different from the subgroup that has a good SSH.

\section{Acknowledgments}

We would like to acknowledge the students who filled out the questionnaires.

\section{Author Contributions}

Nama Tal collected all the data and organized it for the statistical analysis. Dorit Shoval and Orna Tzischinsky analyzed the data and wrote the article together.

\section{Competing Interests}

The authors have declared that no competing interests exist.

\section{References}

1. Papaconstantinou E, Bartfay WJ, Bartfay E. Smartphone use, sleep quality and quantity, and mental health outcomes in a university population. Sleep Med. 2017; 40: e251.

2. IDC Corporate USA. International Data. First Quarter Report 2017 [Internet]. 2017. Available from: https://www.idc.com/getdoc.jsp?containerld=prUS42507917.

3. Demirci K, Akgönül M, Akpinar A. Relationship of smartphone use severity with sleep quality, depression, and anxiety in university students. J Behav Addict. 2015; 4: 85-92.

4. Wolniewicz CA, Tiamiyu MF, Weeks JW, Elhai JD. Problematic smartphone use and relations with negative affect, fear of missing out, and fear of negative and positive evaluation. Psychiatry Res. 2018; 262: 618-623.

5. Kenney EL, Gortmaker SL. United States adolescents' television, computer, videogame, smartphone, and tablet use: Associations with sugary drinks, sleep, physical activity, and obesity. J Pediatr. 2017; 182: 144-149.

6. Banjanin N, Banjanin N, Dimitrijevic I, Pantic I. Relationship between internet use and depression: Focus on physiological mood oscillations, social networking and online addictive behavior. Comput Hum Behav. 2015; 43: 308-312.

7. Selvaganapathy K, Rajappan R, Dee TH. The effect of smartphone addiction on craniovertebral angle and depression status among university students. Int J Integr Med Sci. 2017; 4: 537-542.

8. Scott H, Woods HC. Fear of missing out and sleep: Cognitive behavioural factors in adolescents' 
nighttime social media use. J Adolesc. 2018; 68: 61-65.

9. Kloss JD, Nash CO, Walsh CM, Culnan E, Horsey S, Sexton-Radek K. A “Sleep 101" program for college students improves sleep hygiene knowledge and reduces maladaptive beliefs about sleep. Behav Med. 2016; 42: 48-56.

10. Peltzer K, Pengpid S. Nocturnal sleep problems among university students from 26 countries. Sleep Breath. 2015; 19: 499-508.

11. Eliasson $\mathrm{AH}$, Eliasson $\mathrm{AH}$, Lettieri CJ. Differences in sleep habits, study time, and academic performance between US-born and foreign-born college students. Sleep Breath. 2017; 21: 529533.

12. Cohen A, Abu NB, Haimov I. The interplay between tobacco dependence and sleep quality among young adults. Behav Sleep Med. 2018; 18: 164-176.

13. Suen LK, Tam WW, Hon KL. Association of sleep hygiene-related factors and sleep quality among university students in Hong Kong. Hong Kong Med J. 2010; 16: 180-185.

14. Martin CA, Hiscock H, Rinehart N, Heussler HS, Hyde C, Fuller-Tyszkiewicz M, et al. Associations between sleep hygiene and sleep problems in adolescents with ADHD: A cross-sectional study. J Atten Disord. 2020; 24: 545-554.

15. Mindell JA, Meltzer LJ, Carskadon MA, Chervin RD. Developmental aspects of sleep hygiene: Findings from the 2004 national sleep foundation sleep in America poll. Sleep Med. 2009; 10: 771-779.

16. Rogers AP, Barber LK. Addressing FoMO and telepressure among university students: Could a technology intervention help with social media use and sleep disruption? Comput Hum Behav. 2019; 93: 192-199.

17. Adams SK, Williford DN, Vaccaro A, Kisler TS, Francis A, Newman B. The young and the restless: Socializing trumps sleep, fear of missing out, and technological distractions in first-year college students. Int J Adolesc Youth. 2017; 22: 337-348.

18. Woods HC, Scott H. \# Sleepyteens: Social media use in adolescence is associated with poor sleep quality, anxiety, depression and low self-esteem. J Adolesc. 2016; 51: 41-49.

19. Rosen L, Carrier LM, Miller A, Rokkum J, Ruiz A. Sleeping with technology: Cognitive, affective, and technology usage predictors of sleep problems among college students. Sleep Health. 2016; 2: 49-56.

20. Przybylski AK, Murayama K, DeHaan CR, Gladwell V. Motivational, emotional, and behavioral correlates of fear of missing out. Comput Hum Behav. 2013; 29: 1841-1848.

21. Vorderer P, Krömer N, Schneider FM. Permanently online-Permanently connected: Explorations into university students' use of social media and mobile smart devices. Comput Hum Behav. 2016; 63: 694-703.

22. Mastin DF, Bryson J, Corwyn R. Assessment of sleep hygiene using the sleep hygiene index. J Behav Med. 2006; 29: 223-227.

23. Spielberger CD. Manual for the state-trait anxiety inventory (form $Y$ ) ("self-evaluation questionnaire"). Palo Alto: Consulting Psychologists Press; 1983.

24. Buysse DJ, Reynolds II CF, Monk TH, Berman SR, Kupfer DJ. The Pittsburgh sleep quality index: A new instrument for psychiatric practice and research. Psychiatry Res. 1989; 28: 193-213.

25. George D, Mallery M. SPSS for windows step by step: A simple guide and reference. 10th ed. Boston: Pearson; 2010.

26. Hayes AF. Introduction to mediation, moderation, and conditional process analysis: 
Methodology in the social sciences. New York: Guilford publications; 2013.

27. Faul F, Erdfelder E, Lang AG, Buchner A. G* Power 3: A flexible statistical power analysis program for the social, behavioral, and biomedical sciences. Behav Res Methods. 2007; 39: 175191.

28. Chehri A, Khazaie H, Eskandari S, Khazaie S, Holsboer-Trachsler E, Brand S, et al. Validation of the Farsi version of the revised Adolescent Sleep Hygiene Scale (ASHSr): A cross-sectional study. BMC Psychiatry. 2017; 17: 408.

29. Fobian AD, Avis K, Schwebel DC. The impact of media use on adolescent sleep efficiency. J Dev Behav Pediatr. 2016; 37: 9-14.

30. Falbe J, Davison KK, Franckle RL, Ganter C, Gortmaker SL, Smith L, et al. Sleep duration, restfulness, and screens in the sleep environment. Pediatrics. 2015; 135: e367-e375.

31. Monma T, Ando A, Asanuma T, Yoshitake Y, Yoshida G, Miyazawa T, et al. Sleep disorder risk factors among student athletes. Sleep Med. 2018; 44: 76-81.

32. Shoval D, Tal N, Tzischinsky O. Relationship of smartphone use at night with sleep quality and psychological well-being among healthy students: A pilot study. Sleep Health. 2020; 6: 495-497.

33. Coskun S, Muslu GK. Investigation of problematic mobile phones use and fear of missing out (FoMO) level in adolescents. Community Ment Health J. 2019; 55: 1004-1014.

34. Whipps J, Byra M, Gerow KG, Guseman EH. Evaluation of nighttime media use and sleep patterns in first-semester college students. Am J Health Behav. 2018; 42: 47-55.

35. Suh S, Cho N, Zhang J. Sex differences in insomnia: From epidemiology and etiology to intervention. Curr Psychiatry Rep. 2018; 20: 69.

36. Lau WK, Leung MK, Wing YK, Lee TM. Potential mechanisms of mindfulness in improving sleep and distress. Mindfulness. 2018; 9: 547-555.

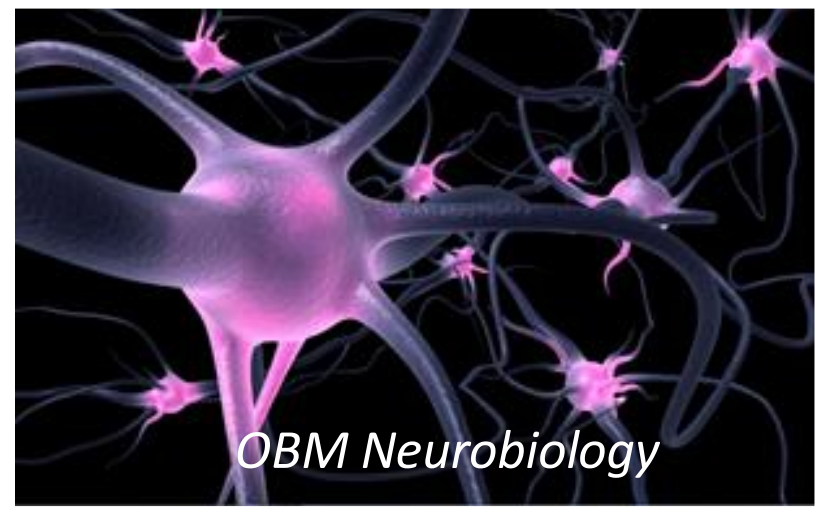

Enjoy OBM Neurobiology by:

1. Submitting a manuscript

2. Joining volunteer reviewer bank

3. Joining Editorial Board

4. Guest editing a special issue

For more details, please visit:

http://www.lidsen.com/journals/neurobiology 